The University of San Francisco

USF Scholarship: a digital repository @ Gleeson Library | Geschke Center

Nursing and Health Professions Faculty

Research and Publications

School of Nursing and Health Professions

$9-26-2018$

The Emerging Role of Neurodiagnostic Informatics in Integrated

Neurological and Mental Health Care

William Bosl

Follow this and additional works at: https://repository.usfca.edu/nursing_fac

Part of the Medicine and Health Sciences Commons 


\section{The Neurodiagnostic Journal}

\section{The Emerging Role of Neurodiagnostic Informatics in Integrated Neurological and Mental Health Care}

\section{William J. Bosl}

To cite this article: William J. Bosl (2018) The Emerging Role of Neurodiagnostic Informatics in Integrated Neurological and Mental Health Care, The Neurodiagnostic Journal, 58:3, 143-153, DOI: 10.1080/21646821.2018.1508983

To link to this article: https://doi.org/10.1080/21646821.2018.1508983

册 Published online: 26 Sep 2018.

Submit your article to this journal

III Article views: 867

View Crossmark data $\nearrow$ 


\title{
The Emerging Role of Neurodiagnostic Informatics in Integrated Neurological and Mental Health Care
}

\author{
William J. Bosl, Ph.D., ${ }^{1,2}$ \\ ${ }^{1}$ Health Informatics and Clinical Psychology Programs \\ University of San Francisco \\ San Francisco, California \\ ${ }^{2}$ Computational Health Informatics Program \\ Boston Children's Hospital \\ Boston, Massachusetts \\ ${ }^{3}$ Department of Pediatrics \\ Harvard Medical School \\ Boston, Massachusetts
}

\begin{abstract}
Mental, neurological, and neurodevelopmental (MNN) disorders impose an enormous burden of disease globally. Many MNN disorders follow a developmental trajectory. Thus, defining symptoms of MNN disorders may be conceived as the end product of a long developmental process. Many pharmaceutical therapies are aimed at the end symptoms, essentially attempting to reverse pathological brain function that has developed over a long time. A new paradigm is needed to leverage the developmental trajectory of MNN disorders, based on measuring brain function through the life span. Electroencephalography (EEG) is ideally suited for this task. New developments in several fields, including consumer EEG hardware, ubiquitous access to the Internet and electronic health records, and nonlinear mathematics to extract information from physiological signals have converged to enable new approaches to integrating EEG into routine health care. Research continues to demonstrate that EEG analysis can be used to discover digital biomarkers for a wide range of MNN disorders, including autism, attention-deficit/hyperactivity disorder (ADHD), schizophrenia and dementias, and likely many others. When EEGderived information about brain function is stored with an electronic health record, clinical decision support software may use these data to detect atypical brain development in the earliest stages, thus opening a potential window for early intervention. These developments create an opportunity
\end{abstract}


for neurodiagnostics to merge with biomedical informatics to create clinical tools for monitoring brain function through the life span. Advanced professionals with neurodiagnostics and biomedical informatics skills and training are needed to lead the way in this emerging field.

KEY WORDS. Biomedical informatics, EEG, mental health, neurodiagnostics, neuroscience.

\section{INTRODUCTION}

Mental, neurological, and neurodevelopmental (MNN) disorders, or simply "brain disorders," impose the largest health burden on the human population of all chronic disease classes in the world (Prince et al. 2007; Idro et al. 2010; Collins et al. 2011). Two different measures of disease burden may be used to demonstrate the tremendous impact of brain disorders on population health. Mental illness alone has been estimated to represent $32.4 \%$ of years lived with disability (YLDs) and $13.0 \%$ of disabilityadjusted life-years (DALYs), both of which are greater than previous estimates by onethird. These estimates place mental illness first in global burden of disease in terms of YLDs and level with cardiovascular and circulatory diseases in terms of DALYs (Vigo et al. 2016). These figures are surprising to most people. Surely mental illness cannot be as much of a health burden as heart disease or cancer. Part of the reason that they impose such a large lifetime burden is that many begin early in life, imposing many decades of disability on the patient (Insel 2014). Brain disorders that are traditionally labeled as neurological disorders rather than mental disorders, such as Alzheimer's disease and Parkinson's disease, add significantly to the global burden of brain disorders.

Yet, the overall health burden of MNN disorders may still be underestimated because current measures fail to take into account the connectedness of mind and body. Many chronic medical conditions have comorbid mental and cognitive conditions leading to poor outcomes. Similarly, mental conditions can cause a number of poor health outcomes, creating a range of serious chronic diseases that require medical care. As the World Health Organization (WHO) constitution states: "Health is a state of complete physical, mental and social well-being and not merely the absence of disease or infirmity." Thus, there is no health without mental health. The lack of research focus given to this enormous health problem by governments and health care organizations has been called a "failure of humanity" (Kleinman 2009). One of the great challenges is how to integrate mental health care into general medical practice, particularly when resources for even more comprehensive health care in both wealthy and poor regions of the world are limited. 
As the average age of the world's population continues to increase, neurodegenerative diseases such as Alzheimer's disease or other dementias and Parkinson's disease exact an increasing toll on individuals, their families, and the health care systems of all nations. Pharmaceutical companies spend enormous amounts of money searching for drugs to reverse the symptoms of neurogenerative disorders, such as Alzheimer's disease and other dementias, but results thus far have been dismal (Mehta et al. 2017). This is not surprising when the developmental trajectory of these disorders is considered. For example, the characteristic motor signs of Parkinson's disease begin to appear only after $70-80 \%$ of the dopamine-producing neurons of the substantia nigra have been lost. By the time symptoms appear, considerable brain degeneration has already occurred. Reducing the symptoms would thus require essentially regenerating large portions of neural tissue that has been lost over many years or decades. Like many mental disorders, symptoms of neurodegenerative diseases are the final end stage of a very long physiological process. Reversing symptoms at this point is tantamount to repairing and reversing extensive neural damage. Perhaps recognizing the challenge, Pfizer, one of the leading pharmaceutical research companies in the world, announced in 2017 that the company would halt research on Alzheimer's and Parkinson's disease drugs, citing disappointing results in recent years (https://perma.cc/4WDX-8XHC).

Evidence continues to accumulate that many, if not all, mental and neurological disorders result from a long neurodevelopmental process that is driven by complex interplay among genetics, early infectious insults, diet, environmental stimuli, and as yet unknown factors. From a clinical perspective, the implication is that most current interventions are attempting to reverse changes that have happened in the brain over a long period of time. Some of these changes may be irreversible once they have occurred. A new paradigm is needed that views health from an integrated perspective. Physiological, neurophysiological, and mental health are all aspects of the integrated human person.

\section{EEG AS A GENERAL TOOL FOR MONITORING BRAIN FUNCTION}

Measurements of brain electrical activity with EEG have long been a valuable source of information for neuroscience research, yet this rich resource may be underutilized for clinical applications in neurology and psychiatry (Niedermeyer 2003; Niedermeyer and Lopes da Silva 2005). At this time, neurodiagnostics is primarily a specialist discipline, focused on tertiary care in epilepsy centers, sleep clinics, intensive and critical care units, and interoperative neurophysiological monitoring. The future of neurodiagnostics requires integration of functional brain measurement and monitoring into routine primary health care. This has not been feasible until recently. Several technical developments are emerging simultaneously that create tremendous new opportunities for neurodiagnostics to address the broad class of MNN disorders in 
the context of routine primary care, opening new opportunities for early intervention. These include new, easy-to-use EEG devices, the widespread use of electronic medical records, the emergence of machine learning and "big-data" analytics, and applications of complex systems theory to cognitive neuroscience. Together, these developments have created the possibility of using EEG as a functional brain monitoring tool in routine health care.

\section{New-Generation EEG Devices}

A new generation of portable, relatively easy to use EEG devices has arisen in the past decade. Some of the early devices were aimed at the computer game industry, with hopes that game developers would incorporate "mind control" into the games. For this to be viable, the EEG devices had to be inexpensive enough for large populations of children and adolescents to buy them, and they had to be very easy to use. Although the mind-controlled computer game market has not taken off as some may have hoped, it did cause a great deal of research to create better-quality, lower-cost EEG sensors and electronics. At least half a dozen consumer EEG devices are now available, with varying levels of quality. Slightly more expensive, medical-grade devices are now coming into the market. The primary significance of these new devices is that they create the possibility of a functional brain measurement in the context of primary care checkups. No other brain measurement technology makes this possible. With health care costs continuing to rise, the time allotted for routine checkups decreasing as physicians become busier, and insurance reimbursements increasingly scrutinized, at this time only EEG has any chance of being used as a functional brain measurement device in routine, integrated health care.

\section{Electronic Health Records and Biomedical Informatics}

Primary care clinicians, whether physicians, clinical psychologists, or nurse practitioners, cannot visually make use of EEG measurements in the same way that trained neurologists read EEGs to detect epileptiform activity or other known pathologies. As will be discussed, early detection of many MNN disorders will require computer algorithms to detect pathologies that visual inspection cannot detect. Furthermore, monitoring changes in brain function requires that data be saved over time with the patient's electronic record. Current electronic health records are not adequately designed to store short EEG segments collected for routine brain monitoring or the digital biomarkers that are derived from them using computational algorithms.

In order for EEG-derived digital biomarkers to become useful for assessing the risk of MNN disorders, biomedical ontologies must continue to be developed for all MNN disorders. Biomedical ontologies are formal descriptions of health-related entities, based in reality, and the relationships between those entities (Smith and 
Scheuermann 2011). Thus, ontologies for MNN disorders represent the constructs that define these disorders and their relationships to all other health concepts. Once properly formulated, in a suitable formal language, the ontologies can be used for sophisticated automated reasoning applications (Hastings et al. 2012; Ceusters et al. 2017). Even for epilepsy, which is generally considered a neurological and thus medical disorder, many of the epilepsy-relevant medical concepts are not yet available in existing biomedical ontologies and therefore not fully represented in electronic health records (Maldonado et al. 2017). Applied research to develop biomedical ontologies for mental illness continues to be active (Ceusters and Smith 2010), and much work is yet to be done to integrate mental health constructs with other medical concepts. Incorporation of EEG-derived features and constructs will be required for incorporating newly discovered EEG biomarkers into routine practice. Professionals with advanced training in clinical neuroscience, biomedical informatics, and neurodiagnostics can contribute a great deal to this work.

\section{Complex Systems Theory and Neuroscience}

Important advances in the use of EEG data for assessing brain function have come from deeper understanding of complex systems theory and its application to neural function. Dynamical systems theory or complex systems theory, sometimes also called chaos theory, is a branch of mathematical physics that deals with the qualitative and quantitative characterization of long-term properties of complex dynamical systems (Bar-Yam, 2000; Pikovsky and Maistrenko 2003; Stam 2005). The brain is a complex dynamical system. This has important implications for understanding the relationship between observed behaviors or cognitive function and the neurophysiology of the brain, which is the locus of every behavior, cognitive phenotype, neuropathology, and psychopathology that is observed. The brain is also an adaptive evolving complex system, implying that its dynamical properties can change over long time periods in response to learning, development, injury, or disease. Before any clinical signs or symptoms of neurological or psychiatric disease are observed, the brain must first change.

Much of neuropsychology and biological psychiatry research has been focused on localizing brain function. Although the effort to map specific brain regions with specific behavioral and cognitive deficits has been clinically useful, many higherlevel cognitive functions have defied localization. For example, recent evidence demonstrates that many neurons in higher-order brain regions such as the prefrontal cortex (PFC) are not organized anatomically. Rather, they are said to exhibit mixed selectivity to multiple aspects of cognitive function (Rigotti et al. 2013). Another way of stating this is that the brain functions as a complex system. If the brain is viewed as a dynamical system, the connectivity pattern between neurons is an important determinant of brain function. Mental and neurological disorders are associated with 
abnormal brain connectivity that may vary between different regions and different scales (Noonan et al. 2009). Estimation of neural connectivity variation or differences might be a useful way to detect abnormal brain function as compared with normal function. The challenge, then, is how to measure changes in brain connectivity that are associated with clinical signs and symptoms of disease that are of interest. Further details can be found in Bosl (2017).

Important theorems in mathematics were proved in the 1980s and 1990s regarding what is known as the reconstruction problem. The embedding or reconstruction theorems of dynamical systems assert that dynamical properties of a complex system, such as the brain, are embedded in the time-series measurements of the components of the system. This has potentially profound implications for EEG analysis. It implies that the scalp measurements of an EEG sensor, which are linear combinations of the contribution of many neurons, contain information about all neurons in the system. This does not mean that all the information about all neurons can be extracted from a finite, discrete time-series measurement. It does imply that brain function of the entire brain can be reconstructed from scalp EEG measurements (Kantz and Schreiber 2004; Huffaker et al. 2017). Thus, the oft-cited charge that EEG measurements only contain information about surface cortical neurons is not correct. Computational methods that implement the reconstruction theorems may thus be used to compute values that represent the dynamical properties of the brain. In principle, the values that represent dynamical properties of a complex system, sometimes called "dynamical invariants," contain information that may distinguish normal and pathological brain function. Although our scientific understanding of the relationship between neurophysiological dynamics and the observed clinical behaviors that define neurological and mental disorders is in its infancy, empirical methods may be used to find clinically useful relationships. This data-driven approach has emerged with the development of big-data analytics.

\section{Biomedical Informatics and Big-Data Analytics}

We accept as an axiom that all observed clinical behaviors of relevance to neurology and psychiatry are reflections of brain activity. An empirical approach to discovering the relationship between measurable brain states and behavior is to simply collect EEG measurements and clinical assessments from a large number of patients with a particular disease or characteristic of interest, and an equally large number of controls with whom to compare. Machine learning or pattern classification algorithms can be used to discover the complicated patterns within the large number of nonlinear features computed from the EEG signals that correlate to the disorder of interest. Technical details for these data-driven approaches are beyond the scope of this article. Instead, we illustrate the process with an example of a recently published method for 
discovering digital biomarkers for autism spectrum disorder (ASD) long before the symptoms of autism appear.

A prospective study of infants at high risk for developing ASD was conducted at Boston Children's Hospital. The following results are reported in Bosl et al. (2018). Autism spectrum disorder (ASD) is a complex and heterogeneous disorder, diagnosed on the basis of behavioral symptoms during the second year of life or later. Evidence continues to mount to suggest that atypical neurodevelopment precedes emergence of the behaviors that define ASD by months or even years. That is, atypical neural function and development occurs long before the behavioral symptoms that define ASD emerge. If changes in the brain can be detected when they first begin to occur, a critical window for early intervention might exist. This has fueled a search for early neural correlates or neurobiological indicators that precede the emergence of ASD, which typically isn't diagnosed clinically until after 3 years of age.

EEG measurements were collected from 99 high-risk infants with an older sibling diagnosed with ASD, and 89 low-risk controls, beginning at 3 months of age and continuing until 36 months of age. Nonlinear features derived from the reconstruction methods described previously were computed from EEG signals and used as input to statistical learning methods. Pattern classifiers or statistical learning algorithms (specifically, a support vector machine or SVM) were used to find the mapping from the nonlinear values to the clinical outcome. Prediction of the 3-year clinical diagnostic outcome of ASD or not ASD was highly accurate from as early as 3 months of age, demonstrating that useful digital biomarkers might be extracted from EEG measurements.

There is evidence that quantitative analysis of EEG data using this data-driven approach may be possible for early detection of a number of other MNN disorders, including epilepsy (Bosl et al. 2017), attention-deficit/hyperactivity disorder (ADHD) (Snyder et al. 2015; Gloss et al. 2016), schizophrenia (Li et al. 2008; Raghavendra et al. 2009; Takahashi et al. 2010), Alzheimer's disease (Abasolo et al. 2006; Knyazeva et al. 2013), depression ( $\mathrm{Li}$ et al. 2008; Gordon et al. 2010), and others. In principle, when nonlinear values extracted from EEG signals are monitored over time using computer algorithms, deviations that indicate the emergence of many pathological conditions should be detectable. Empirical research with clinical data will be required to demonstrate this.

Application of neurodiagnostics in primary care screening requires a different approach from current neurodiagnostic centers. This will not replace current EEG centers but create an entirely new and different application. To be useful, quick EEG scans taken in a primary care setting must be saved with the patient's electronic health record. Computational algorithms must compute relevant information from the signals and construct developmental trajectories for the patient, monitoring changes over time and comparing with large normative databases. This will allow risk assessment information, not definitive diagnosis. This information becomes useful 
to a range of health care providers: primary care physician, psychologist, social worker, nurse practitioner, as well as specialists in neurology and psychiatry, among others. Early risk assessment will enable our providers to implement preventive therapies to reduce or eliminate the risk of disease progression. All of this of course requires further research.

\section{NEXT STEPS}

The simultaneous emergence of portable, efficient EEG hardware, electronic medical records, nonlinear methods for analyzing complex systems, and systems neuroscience has created a remarkable opportunity for creation of a new paradigm for early detection and treatment of mental, neurological, and neurodevelopmental disorders. The combination of skills that will be needed to take these disparate research results to the clinic as a unified clinical support technology for practicing clinicians includes neurodiagnostics, neurology, psychiatry, and biomedical informatics. An opportunity is emerging for the next generation of neurodiagnostic technologists to obtain advanced training in biomedical informatics and clinical neurophysiology, applying this integrated, advanced training to the creation of digital biomarkers to monitor the brain for psychiatric and neurological disorders. This new field is still awaiting definition but may be called "neuroinformatics" or "neurodiagnostic informatics," indicating the critical integration of neurotechnology, clinical neuroscience, and information technology. This is a natural evolution of EEG technology with biomedical informatics and information technology. Before the genomic revolution was possible, mathematical and computational tools for analyzing nucleotide sequences from DNA were required to make sense of the new data source. Similarly, computational methods for analyzing EEG signals will be required to make EEG fully useful for new applications.

There is a precedent for a nonphysician, advanced-level certification. Physicians who are board certified in a primary medical specialty may become board certified in clinical informatics as a subspecialty by completing an accredited, 2-year fellowship in clinical informatics and passing a written examination. A parallel certification for nonphysician professionals will soon be available, an Advanced Health Informatics Certification (AHIC), which has been led by the American Medical Informatics Association (AMIA; www.amia.org). AMIA previously led the successful effort to create the clinical informatics subspecialty for American Board of Medical Specialties board-certified physicians. Similar in rigor to the physicians' board certification, AHIC requires a master's degree in health or biomedical informatics and a master's or doctorate in a clinical or health care field (Fridsma 2016; Gadd et al. 2016a, 2016b).

A certification for advanced-level neurodiagnostic professionals might require certification in one or more current neurodiagnostic technology specialties, a master's degree in neurodiagnostics or clinical neuroscience, and perhaps even a doctorate in a clinical neuroscience or clinical neurodiagnostics field. Graduate programs in 
neurodiagnostics, neurodiagnostic informatics, or clinical neuroscience may need to be created. A doctorate in clinical neurophysiology, similar to professional doctorates in other health care fields, with an appropriately defined scope of practice, should be a goal for the neurodiagnostics community. The author is working with leadership to implement a master's degree program in neurodiagnostic informatics at the University of San Francisco that will combine graduate-level training in all of the disciplines described here. Interested neurodiagnostic professionals and neurophysiologists with an interest in such a program, as either students or faculty, are encouraged to contact the author directly by email.

ASET-The Neurodiagnostic Society reports a significant shortage of neurodiagnostic technologists to fill current needs (ASET 2016). The need for neurodiagnostic technologists is likely to continue to increase, as applications of EEG in intensive and critical care units will require neurotechnologists with advanced training to screen EEG data to support physicians with neurophysiology training who are often under tremendous time pressure. The new applications of EEG to primary care brain monitoring represent a new field where neurotechnologists may contribute. Since this subfield does not yet exist, we can only speculate about what role neurotechnologists might play. Initially, neurotechnologists with graduate-level training as described in this article will work alongside clinical informaticists to help implement protocols and data procedures within electronic medical record systems. The need for health data scientists continues to grow tremendously, and those with training in both biomedical informatics and specialty knowledge in neurology, neuropsychology, and neurodiagnostics will make unique contributions to emerging integrated health care endeavors.

The neurodiagnostics, neurophysiology, and medical communities will have to come together to agree upon standards for an advanced level of certification for neurodiagnostic professionals and the scope of practice for these professionals. The scope of practice might include authority to independently read EEGs, knowledge and skill to implement clinical databases, create ontologies for electronic health records, and implement analytics software for clinical decision support systems that use EEGderived data. Although this must be determined by the relevant medical and clinical governing boards, it is clear that the current shortage of neurodiagnostic professionals, and the need for an advanced education and career path to meet growing needs in this field, will help to define the exciting future field of neurodiagnostics and neurodiagnostic informatics.

\section{REFERENCES}

Abasolo D, Hornero R, Espino P, Alvarez D, Poza J. 2006. Entropy analysis of the EEG background activity in Alzheimer's disease patients. Physiol Meas. 27:241-253.

ASET. 2016. Editorial: building a qualified neurodiagnostic technology workforce. Neurodiagn J. $56: 131-138$.

Bar-Yam Y. 2000. Unifying themes in complex systems. Boulder (CO): Westview Press. 
Bosl W. 2017. Chapter 14: EEG-derived neurophenotypes. In: Jagaroo V, Santangelo S, editors. Neurophenotypes: advancing psychiatry and neuropsychology in the "OMICS" era. New York (NY): Springer.

Bosl WJ, Loddenkemper T, Nelson CA. 2017. Nonlinear EEG analysis reveals commonalities and differences in autism and absence epilepsy. Neuropsychiatr Electrophysiol. 3:1. doi:10.1186/s40810-017-0023-x.

Bosl WJ, Tager-Flusberg H, Nelson CA. 2018. EEG analytics for early detection of autism spectrum disorder: a data-driven approach. Sci Rep. 8:6828.

Ceusters W, Jensen M, Diehl AD. 2017. Ontological realism for the research domain criteria for mental disorders. Stud Health Technol Inform. 235:431-435.

Ceusters W, Smith B. 2010. Foundations for a realist ontology of mental disease. J Biomed Semantics. 1:10.

Collins PY, Patel V, Joestl SS, March D, Insel TR, Daar AS, Anderson W, Dhansay MA, Phillips A, Shurin S, et al. 2011. Grand challenges in global mental health. Nature. 475:27-30.

Fridsma DB. 2016. The scope of health informatics and the Advanced Health Informatics Certification. J Am Med Inform Assoc. 23:855-856.

Gadd CS, Williamson JJ, Steen EB, Andriole KP, Delaney C, Gumpper K, LaVenture M, Rosendale D, Sittig DF, Thyvalikakath T, et al. 2016a. Eligibility requirements for advanced health informatics certification. J Am Med Inform Assoc. 23:851-854.

Gadd CS, Williamson JJ, Steen EB, Fridsma DB. 2016b. Creating advanced health informatics certification. J Am Med Inform Assoc. 23:848-850.

Gloss D, Varma JK, Pringsheim T, Nuwer MR. 2016. Practice advisory: the utility of EEG theta/beta power ratio in ADHD diagnosis: report of the Guideline Development, Dissemination, and Implementation Subcommittee of the American Academy of Neurology. Neurology. 87:2375-2379.

Gordon E, Palmer DM, Cooper N. 2010. EEG alpha asymmetry in schizophrenia, depression, PTSD, panic disorder, ADHD and conduct disorder. Clin EEG Neurosci. 41:178-183.

Hastings J, Smith B, Ceusters W, Jensen M, Mulligan K (2012). Representing mental functioning: ontologies for mental health and disease. ICBO 2012: 3rd International Conference on Biomedical Ontology (Citeseer).

Huffaker R, Bittelli M, Rosa R. 2017. Nonlinear time series analysis with R. New York (NY): Oxford University Press.

Idro R, Newton C, Kiguli S, Kakooza-Mwesige A. 2010. Child neurology practice and neurological disorders in East Africa. J Child Neurol. 25:518-524.

Insel TR. 2014. Mental disorders in childhood: shifting the focus from behavioral symptoms to neurodevelopmental trajectories. JAMA. 311:1727-1728.

Kantz H, Schreiber T. 2004. Nonlinear time series analysis. 2nd ed. New York (NY): Cambridge University Press.

Kleinman A. 2009. Global mental health: a failure of humanity. Lancet. 374:603-604.

Knyazeva MG, Carmeli C, Khadivi A, Ghika J, Meuli R, Frackowiak RS. 2013. Evolution of source EEG synchronization in early Alzheimer's disease. Neurobiol Aging. 34:694-705.

Li Y, Tong S, Liu D, Gai Y, Wang X, Wang J, Qiu Y, Zhu Y. 2008. Abnormal EEG complexity in patients with schizophrenia and depression. Clin Neurophysiol. 119:1232-1241.

Maldonado R, Goodwin TR, Skinner MA, Harabagiu SM. 2017. Deep learning meets biomedical ontologies: knowledge embeddings for epilepsy. AMIA Annu Symp Proc. 2017:1233-1242.

Mehta D, Jackson R, Paul G, Shi J, Sabbagh M. 2017. Why do trials for Alzheimer's disease drugs keep failing? A discontinued drug perspective for 2010-2015. Expert Opin Investig Drugs. 26:735-739.

Niedermeyer E. 2003. The clinical relevance of EEG interpretation. Clin Electroencephalogr. 34:93-98.

Niedermeyer E, Lopes da Silva FH. 2005. Electroencephalography: basic principles, clinical applications, and related fields. 5th ed. Philadelphia (PA): Lippincott Williams \& Wilkins.

Noonan SK, Haist F, Muller RA. 2009. Aberrant functional connectivity in autism: evidence from low-frequency BOLD signal fluctuations. Brain Res. 1262:48-63.

Pikovsky A, Maistrenko IUL. 2003. Synchronization: theory and application. Dordrecht; Boston (MA): Kluwer Academic Publishers.

Prince M, Patel V, Saxena S, Maj M, Maselko J, Phillips MR, Rahman A. 2007. No health without mental health. Lancet. 370:859-877.

Raghavendra BS, Dutt DN, Halahalli HN, John JP. 2009. Complexity analysis of EEG in patients with schizophrenia using fractal dimension. Physiol Meas. 30:795-808. 
Rigotti M, Barak O, Warden M, Wang XJ, Daw N, Miller E, Fusi S. 2013. The importance of mixed selectivity in complex cognitive tasks. Nature. 497:585-590.

Smith B, Scheuermann RH. 2011. Ontologies for clinical and translational research: introduction. J Biomed Inform. 44:3-7.

Snyder SM, Rugino TA, Hornig M, Stein MA. 2015. Integration of an EEG biomarker with a clinician's ADHD evaluation. Brain Behav. 5:e00330.

Stam CJ. 2005. Nonlinear dynamical analysis of EEG and MEG: review of an emerging field. Clin Neurophysiol. 116:2266-2301.

Takahashi T, Cho RY, Mizuno T, Kikuchi M, Murata T, Takahashi K, Wada Y. 2010. Antipsychotics reverse abnormal EEG complexity in drug-naive schizophrenia: a multiscale entropy analysis. Neuroimage. $51: 173-182$.

Vigo D, Thornicroft G, Atun R. 2016. Estimating the true global burden of mental illness. Lancet Psychiatry. $3: 171-178$. 